
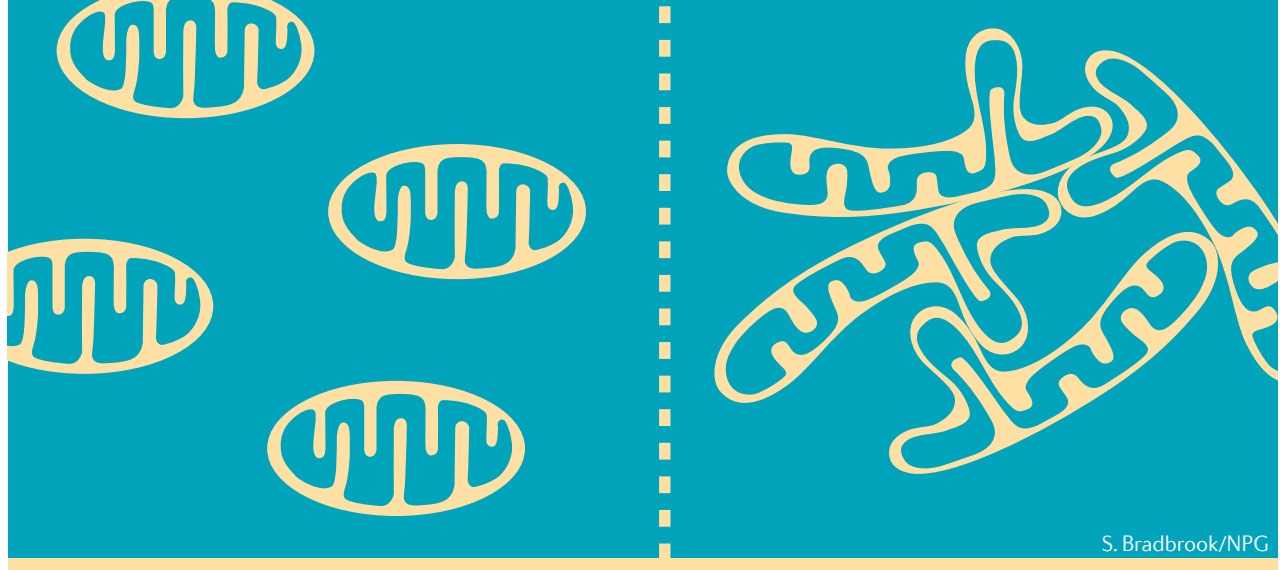

T CELLS

\title{
Mitochondrial shape shifters
}

A study recently published in Cell shows that the change in metabolic activity that occurs as $\mathrm{T}$ cells differentiate into effector cells or memory cells is controlled by structural remodelling of their mitochondria. Punctate mitochondria with loosened cristae in effector T cells promote anabolic pathways of metabolism, such as aerobic glycolysis, whereas fused mitochondria in memory $\mathrm{T}$ cells engage catabolic pathways, such as fatty acid oxidation. The authors show that forcing mitochondrial fusion in effector $\mathrm{T}$ cells could be used therapeutically to improve $\mathrm{T}$ cell metabolic fitness.

Mitochondria constantly remodel their structure to support metabolic activity, antiviral responses and cell death. Erika Pearce and colleagues investigated whether mitochondrial remodelling might control metabolic adaptations in T cells. Ultrastructural analysis by electron microscopy revealed that effector $\mathrm{T}$ cells have small distinct mitochondria (an indication of mitochondrial fission), whereas memory $\mathrm{T}$ cells have densely packed fused mitochondria. Accordingly, the mitochondrial fission factor dynamin-related protein 1 was more active in effector $\mathrm{T}$ cells than memory $\mathrm{T}$ cells and the mediators of mitochondrial fusion mitofusin 2 and optic atrophy 1 (OPA1) were more highly expressed by memory T cells than effector T cells. T cells lacking OPA1 had a marked survival defect in culture conditions that promote memory $\mathrm{T}$ cells and showed less oxidative phosphorylation (OXPHOS) activity and more glycolytic activity compared with normal cells. Moreover, when transferred into wild-type mice, Opa1 $1^{-/-} \mathrm{T}$ cells failed to generate memory $\mathrm{T}$ cells after infection, although the effector response was normal, which suggests that OPA 1 is required for the generation of memory $\mathrm{T}$ cells but not effector T cells.

To test whether OPA1-mediated mitochondrial fusion supports the metabolism needed for memory $\mathrm{T}$ cell development, the authors cultured effector $\mathrm{T}$ cells with drugs that inhibit fission or promote fusion. The drug-treated effector $\mathrm{T}$ cells had enhanced mitochondrial fusion and acquired memory $\mathrm{T}$ cell properties, such as an increase in OXPHOS over glycolysis and expression of memory $\mathrm{T}$ cell markers such as CD62L and CD127.
Improving $\mathrm{T}$ cell fitness through ex vivo culture is a key goal in the design of adoptive cellular immunotherapies, so the authors tested whether fusion-promoting drugs could be used to create metabolically fit $\mathrm{T}$ cells. Indeed, drug-treated effector $\mathrm{T}$ cells showed better survival following transfer into congenic mice and generated a more robust response to infection. In addition, mice that received drug-treated T cells were able to control tumour growth better than mice that received control-treated T cells.

So, how might OPA1 support memory T cell metabolism? As well as promoting fusion of the inner mitochondrial membrane, OPA1 mediates cristae remodelling. Mitochondrial cristae in effector $\mathrm{T}$ cells were found to be more loosely organized than those in memory $\mathrm{T}$ cells. So the authors suggested that this disorganization leads to the dissociation of electron transport chain (ETC) complexes and to less efficient ETC and OXPHOS activity. Inefficient ETC activity may cause electrons to linger and NADH levels to increase, as seen in effector T cells. To restore redox balance, effector T cells must increase aerobic glycolysis and export lactate to regenerate $\mathrm{NAD}^{+}$ from cytosolic $\mathrm{NADH}$, enforcing their metabolic phenotype. OPA1 may function to prevent this shift to aerobic glycolysis and maintain metabolic quiescence in memory $\mathrm{T}$ cells by tightening cristae complexes together for efficient OXPHOS.

So, mitochondrial fission and fusion regulate cristae remodelling, which alters ETC efficiency and redox balance, and ultimately controls metabolic programming in T cells.

Lucy Bird

ORIGINAL ARTICLE Buck, M. D. et al.

Mitochondrial dynamics controls $T$ cell fate through metabolic programming. Cell http://dx.doi.org/10.1016/j.cell.2016.05.035 (2016) FURTHER READING Man, K. \& Kallies, A. Synchronizing transcriptional control of T cell metabolism and function. Nat. Rev. Immunol. 15 574-584 (2015) 